\title{
ShenmaYizhi Decoction Improves the Mitochondrial Structure in the Brain and Ameliorates Cognitive Impairment in VCl Rats via the AMPK/UCP2 Signaling Pathway
}

\author{
Chengcheng Sun' \\ Meixia Liu (D) \\ Jiangang Liu ${ }^{2}$ \\ Tingting Zhang $\left.\mathbb{D}^{3}\right)^{3}$ \\ Lei Zhang ${ }^{4}$ \\ Hao Li (ID) \\ Zenggang Luo ${ }^{5}$
}

'Department of Geriatrics, Xiyuan Hospital, China Academy of Chinese Medical Sciences, Beijing, I0009I.

People's Republic of China; ${ }^{2}$ Department of Cardiovascular, Xiyuan Hospital, China Academy of Chinese Medical Sciences, Beijing, I0009I, People's Republic of China; ${ }^{3}$ Department of Geriatrics, College of First Clinical Medicine, Shandong University of Traditional Chinese Medicine, Jinan, ShanDong Province, People's Republic of China; ${ }^{4}$ Department of Emergency, Xiyuan Hospital, China Academy of Chinese Medical Sciences, Beijing, I0009I, People's Republic of China; ${ }^{5}$ Medical Administration Office, Beijing Administration of Traditional Chinese Medicine, Beijing, 100053, People's Republic of China
Correspondence: Meixia Liu; Jiangang Liu Email liumeixia2004@126.com;

liujiangang2002@sina.com
Background: ShenmaYizhi decoction (SMYZD) is an effective prescription of traditional Chinese medicine used to treat vascular dementia (VD). Modern research methods have identified its active ingredients clearly as gastrodin, ferulic acid, ginsenosides, and $\beta$ sitosterol. Chronic cerebral hypoperfusion is a driving factor or risk factor for VD, which leads to the disturbance of mitochondrial structure and function.

Purpose: To observe whether SMYZD improves cognitive impairment by improving mitochondrial structure and function.

Methods: Forty adult rats with vascular cognitive impairment (VCI) caused by the bilateral ligation of common carotid arteries were divided into four groups randomly, including the model group, donepezil group, and low-dose and high-dose SMYZD groups, with 10 rats in each group. Additionally, a sham group was established with 10 rats as the control group. The treatment groups were administered donepezil and two different dosages of SMYZD. The donepezil group was administered $0.45 \mathrm{mg} / \mathrm{kg} / \mathrm{d}$ donepezil, and the SMYZ-L group was administered $2.97 \mathrm{~g} / \mathrm{kg} / \mathrm{d}$ SMYZ, which were equivalent to the clinical dosage. The SMYZ-H group was administered $11.88 \mathrm{~g} / \mathrm{kg} / \mathrm{d}$ SMYZ, which is 4 times higher than the clinically equivalent dosage. A shamoperated group was used as the control group and administered an equal volume of distilled water. The rats were treated by gavage for 8 consecutive weeks. Morris water maze (MWM) test was performed to evaluate the learning and memory ability. The mitochondria of brain tissue were extracted from brain for further test. Mitochondrial morphology and the signal path of AMPK/ PPAR $\alpha /$ PGC-1 $\alpha / \mathrm{UCP} 2$ in mitochondria were detected.

Results: With the SMYZD intervention, behavioral performance of rats and pathological changes of mitochondria of brain tissue were significantly improved. In the serum, SOD, GSH-Px, and GSH activities were increased, and the MDA content was decreased. Moreover, the AMPK, PPAR $\alpha$, PGC-1 $\alpha$, UCP2, and ATP5A mRNA and protein expression levels were also reversed by SMYZD. Conclusion: SMYZD may provide a potential therapeutic strategy via activating the AMPK/PPAR $\alpha / \mathrm{PGC}-1 \alpha / \mathrm{UCP} 2$ signal pathway to improve mitochondrial structure and energy metabolism thereby alleviate vascular cognitive impairment.

Keywords: vascular cognitive impairment, ShenmaYizhi decoction, mitochondria energy metabolism, bilateral common carotid artery occlusion

\section{Introduction}

Vascular dementia (VD) is caused by cumulative damage to the brain tissue mediated by vascular factors. It is an acquired, chronic and progressive intellectual 
impairment syndrome that is characterized by gradual impairments in memory and cognitive ability and is widely considered next to Alzheimer's disease (AD) in prevalence among all types of dementia. ${ }^{1,2}$ With the intensification of global ageing, the incidence of dementia is increasing at an alarming rate in all parts of the world, which makes dementia a public health priority. ${ }^{3}$ The main pathogenic mechanism of VD includes chronic cerebral hypoperfusion, ${ }^{4}$ hippocampal neuron loss, ${ }^{5}$ synaptic disorders, ${ }^{6}$ axonal abnormalities, ${ }^{7}$ and white matter vascular changes. ${ }^{8}$ Vascular cognitive impairment (VCI) includes the whole process of vascular cognitive impairment from mild to severe changes, in which vascular pathology changes significantly affects the cognitive status, leading to cognitive impairment and dementia. Insufficient blood perfusion/vascular risk factors enhance memory impairment, accelerate the progression of memory impairment and exert a negative effect on the outcome of treatment. ${ }^{6}$ However, owing to its multiple aetiologies, treatments for VD are limited, and the main clinical treatment for VD is to control risk factors actively and prevent stroke recurrence. ${ }^{9}$

As the center of cell metabolism and the main regulator of redox balance, mitochondria are also the main organelles involved in ischemia, which are very important for the regulation of energy metabolism, signaling pathways and cell death. ${ }^{10}$ The brain is the organ with the most active energy metabolism in the body, and stable and coordinated brain energy metabolism is very important to maintain the normal state of the brain. ${ }^{11}$ Therefore, the proper function of mitochondria is essential for the maintenance of the brain. Evolutionary studies have shown that the emergence of higher cognitive function is related to an increase in glucose utilization and the expression of energy metabolism-related genes. ${ }^{11}$ When the cerebral blood supply is interrupted, the brain cell damage is caused, cognitive function is closely related to energy metabolism. The energy supply is very important in the effective treatment of ischemic diseases. ${ }^{12}$ Based on these findings, dysfunction of brain mitochondria also plays an important role in the pathogenesis of VCI.

AMP-activated protein kinase (AMPK) is generally regarded as a cellular energy sensor and signal sensor of adenine nucleotides that is regulated by various metabolic stresses and plays a major role in regulating the cellular energy balance. ${ }^{13}$ AMPK regulates the content of mitochondria and oxidative stress in neurons and plays an important role in preventing cognitive impairment caused by prenatal stress. ${ }^{14}$ As a ligand-activated transcription factor, PPAR $\alpha$ plays a key role in regulating lipid metabolism, inflammation and energy homeostasis and mediates the improvement in hippocampal synaptic plasticity in transgenic mice with cognitive impairment. ${ }^{15,16}$ PGC$1 \alpha$ is the hub of energy metabolism, plays a key role in mitochondrial biogenesis and energy metabolism and is the main regulator of mitochondrial function. ${ }^{17} \mathrm{UCP} 2$ is an ion transporter located in the intima of mitochondria that participates in maintaining mitochondrial function and regulating the immune response and oxidative stress under physiological and pathological conditions. ${ }^{18}$ According to recent studies, PGC-1 $\alpha$ is involved in the pathophysiology of VD because it increases the expression of mitochondrial antioxidants and uncoupling protein (UCP), improves the energy metabolism of neurons, and provides the possibility for the treatment of cognitive impairment. ${ }^{19}$ The mitochondrial synthetic protease ATP-5A is not only the core hub of mitochondria structure and function but also promotes the formation of the mitochondrial crest. Furthermore, it is also the main centre of cellular homeostasis. $^{20}$

Traditional Chinese medicine has unique advantages in the clinical treatment of VD because of its multi-effect and multi-target characteristics. Shenmayizhi decoction (SMYZD) is composed of Panax ginseng C.A. Mey (Renshen), Gastrodia elata Bl. (Tianma), Euonymus alatus Sieb. (Thunb.) (Guijianyu), and Ligusticum chuanxiong Hort. (Chuanxiong). The main active ingredient of ginseng is ginsenoside, which is a variety of steroidal saponins with the ability to target a variety of tissues and produce various pharmacological reactions. ${ }^{21}$ Ginseng also contains polysaccharides, peptides, polyacetylenic alcohols, fatty acids, etc. ${ }^{22}$ The main chemical constituent of Gastrodia elata $\mathrm{Bl}$. is gastrodin. ${ }^{23}$ Previous studies have reported several biologically active and structurally interesting constituents from Euonymus alatus Sieb. (Thunb.), ${ }^{59}$ in the form of sesquiterpenes, including sesquiterpene alkaloids, triterpenes, flavonoids, and phenolic compounds, such as $\beta$-sitosterol, aromadendrin, and alatamine. ${ }^{24,60}$ Many compounds have been isolated from Ligusticum chuanxiong Hort ${ }^{61}$ including ligustilide, tetramethyl pyrazine (TMP), and ferulic acid. ${ }^{25}$

Previous animal experiments conducted by our research group have shown that SMYZD exerts a significant effect on the treatment of dementia. ${ }^{26-28}$ Nonetheless, the therapeutic mechanism of SMYZD in mitochondrial biogenesis via the AMPK signaling 
pathway remains to be elucidated. Therefore, we investigated the regulation of mitochondrial biogenesis by SMYZD through the AMPK/PPAR $\alpha / \mathrm{PGC}-1 \alpha / \mathrm{UCP} 2$ signaling pathway in the brain tissue of VCI rats to further define the potential mechanism in this study.

\section{Materials and Methods}

\section{Animals}

70 SPF Sprague-Dawley (SD) rats aged 10 weeks, weighing $300 \pm 20 \mathrm{~g}$, half male and half female, were provided by Beijing Vital River Laboratory Animal Technology Co., Ltd., with license No.:SCXK (Beijing) 2016-0006. The rats were giving adaptive feeding in the experimental animal room in the barrier environment with a temperature of $22-26^{\circ} \mathrm{C}$, humidity of $40 \%-70 \%$, and free intake of water and diet, experimental study was carried out one week later. Animal modelling and experiments were performed in strict accordance with the recommendations in the Guide for the Care and Use of Laboratory Animals of the National Institutes of Health. This experiment was approved by the Committee on Ethics of Animal Experiments of Xiyuan Hospital of China Academy of Chinese Medical Sciences (No. 2018XLC004-2).

\section{Chemicals and Reagents}

Haematoxylin-eosin (HE, Fluka (USA)) was imported and packaged separately by Shanghai Chemical Reagent Co., Ltd., batch number: 46160. Donepezil hydrochloride, $5 \mathrm{mg}$ per tablet (trade name: Anlishen) was obtained from Eisai China Inc. (batch number: 1702012). Ginsenoside Rg1 reference substance, purity $96.3 \%$, ginsenoside Re reference substance, 92.9\%, ginsenoside Rb3 reference substance, purity $89.1 \%$, provided by Shanghai Yuanye Biotechnology Co., Ltd.; gastrodin reference substance, purity 98.0\%, 4-p-hydroxybenzyl alcohol, provided by Shanghai Shidande Biotechnology Co., Ltd. The purity of ferulic acid reference substance, is $90.6 \%$, protocatechuic acid reference substance, purity $95.0 \%$, nifedipine/ leaf harringtonine (alatamine) reference substance, purity $90.0 \%$, $\beta$-sitosterol reference substance, purity 98\%, provided by Chengdu Plant Standard Chemical Pure Biotechnology Co., Ltd.

\section{Preparation of SMYZD}

In this experiment, based on the results from the previous stage, ${ }^{62}$ the method for preparing SMYZD was improved, and the preparation process was optimized. Herb slices of SMYZD (composition: Panax ginseng C.A. Mey (Renshen), Gastrodia elata Bl. (Tianma), Euonymus alatus Sieb. (Thunb.) (Guijianyu), and Ligusticum chuanxiong Hort. (Chuanxiong) in a ratio of $3: 3: 3: 2$ ) were prepared in the preparation room of Xiyuan Hospital of China Academy of Chinese Medical Sciences, Beijing Hospital preparation, Beijing Medicine preparation: Z20200005000. Ginseng slices were decocted alone and with water twice: first with 12 times the amount of water for 2 hours and then with 10 times the amount of water for 2 hours. The decoction was filtered and combined. Gastrodia elata Bl. (Tianma), Euonymus alatus Sieb. (Thunb.) (Guijianyu), and Ligusticum chuanxiong Hort. (Chuanxiong) were decocted with water three times using 10 times the amount of water for 2 hours 2 times and 8 times the amount of water for 1 hour 1 time. The filtrate was combined with the ginseng filtrate, and the filtrate was concentrated to produce the extract with a relative density of 1.10 $\pm 1.15\left(50{ }^{\circ} \mathrm{C}\right)$. Each gram of extract contains 2.44 $\mathrm{g}$ of crude drug.

\section{Liquid Chromatography-Mass Spectrometry (LC-MS) Analysis}

The main chemical constituents of SMYZD were analyzed by liquid chromatography-mass spectrometry (LC-MS). The chromatographic column was SymmetryLuna C18 column $(150 \mathrm{~mm} \times 2.1 \mathrm{~mm}, \mathrm{~L} \times$ I.D), 02533103611915 (Waters company, USA), and the pre-column was $0.2 \mathrm{~cm}$ sieve plate, batch number 06222010 (ESA company, USA). The column temperature is $20^{\circ} \mathrm{C}$, the injection volume is $10 \mu \mathrm{L}$, the automatic injector is at room temperature, and the running time is $9.5 \mathrm{~min}$. The mobile phase is aqueous solution containing $0.1 \%$ formic acid, the organic phase is methanol-acetonitrile isobaric mixture (containing $0.1 \%$ formic acid), the flow rate is $0.23 \mathrm{~mL} / \mathrm{min}$, at room temperature, and the injection volume is $5 \mathrm{ul}$. Ginsenoside $\mathrm{Rg} 1$, ginsenoside $\mathrm{Re}$, ginsenoside $\mathrm{Rb} 3$, gastrodin, 4-p-hydroxybenzyl alcohol, ferulic acid, protocatechuic acid and leaf harringtonine (alatamine), $\beta$-sitosterol were precisely weighed and dissolved by ultrasonic with $50 \%$ methanol aqueous solution. And gradually diluted to a solution with a concentration of $5 \mathrm{ug} / \mathrm{mL}$. Pass through $0.22 \mu \mathrm{m}$ filter membrane. 


\section{Animals Model and Drug Intervention}

The method of animal modeling is improved according to the literature. ${ }^{63}$ Rats were fasted for 12 hours before the operation and intraperitoneally anaesthetized with $2 \%$ pentobarbital sodium $(0.2 \mathrm{~mL} / 100 \mathrm{~g})$. Then, rats were fixed in the supine position, the neck skin was disinfected with iodophor and cut $1.5 \mathrm{~cm}$, the subcutaneous tissue and muscle were bluntly separated, and the bilateral common carotid arteries were exposed and ligated. The wound was sutured, and the body temperature of animals was maintained until the animals were able to eat. In the sham operation group, bilateral common carotid arteries were bluntly separated without ligation. Penicillin was administered for 3 days postoperatively at a dose of 40,000 U/day (intramuscular injection) to prevent infection, and wound healing was closely observed. Two weeks later, the Morris water maze test was conducted, and 40 rats with VCI were selected for comparison with the sham operation group. According to their body mass, the rats were randomly divided into 4 groups of 10 rats per group: model control group (Model), donepezil hydrochloride group (Donepezil, $0.45 \mathrm{mg} \cdot \mathrm{kg}-1$ ), SMYZD high-dose group (SMYZ-H, $11.88 \mathrm{~g} \cdot \mathrm{kg}-1$ ), and SMYZD low-dose group (SMYZ-L, $2.97 \mathrm{~g} \cdot \mathrm{kg}-1)$. The dose administered to animals was based on the clinical equivalent dose used in humans. The acute toxicity test of SMYZD was carried out in the research group, and the mice did not produce obvious toxic symptoms. ${ }^{64}$ According to the conversion of human and animal body surface areas, each group was intragastrically administered the same volume of drugs once a day. In addition, 10 rats in the sham operation group (Sham) were intragastrically administered the same volume of pure water. Eight weeks later, the related tests and indexes were detected.

\section{The Morris Water Maze Test}

The Morris water maze test (MWM) was used to detect the spatial learning and memory abilities of rats. The test consisted of place navigation and spatial probe tests. The pool was divided into four quadrants, and a platform was placed in the centre of the first quadrant. After the injection of clean water above the platform $(1 \mathrm{~cm})$, the water temperature was maintained at $21 \pm 2{ }^{\circ} \mathrm{C}$, and an appropriate amount of ink was added to hide the platform. Place navigation experiment: The rats were trained twice a day for a fixed period of time. According to the randomization principle, two different entry points were randomly selected for rats to enter the water, and the rats were gently placed in the water from the entry point facing down to the pool wall. At the same time, the time from entering the water to finding and climbing onto the platform (the escape latency) and swimming distance were recorded. Place navigation was evaluated daily for 4 days. The spatial probe test: The test was conducted 24 hours after the place navigation test. The platform was removed, and the activity time in the first quadrant and the number of times crossing the original platform were recorded. The MWM test operated refers to Cao's method. ${ }^{65}$

\section{Tissue Preparation}

After the Morris water maze test and a 12 hour fast, blood was collected from the abdominal aorta of anesthetized rats, (intraperitoneally injected with $2 \%$ pentobarbital sodium $(0.2 \mathrm{~mL} / 100 \mathrm{~g}))$ and serum was separated on the next day. The related indexes were detected after centrifugation at $4{ }^{\circ} \mathrm{C}$ and $3000 \mathrm{r} / \mathrm{min}$ for $15 \mathrm{~min}$. The brain tissue was quickly and completely removed and randomly divided into groups. The hemispheres of 6 rats in each group were fixed (placed in a $4 \%$ neutral paraformaldehyde solution), and the hemispheres of the other 6 rats were snap-frozen in liquid nitrogen and stored at $-80{ }^{\circ} \mathrm{C}$ until further analysis.

\section{HE Staining}

After fixation, the brain tissue was treated by dehydration, clearing, embedding, slicing, dewaxing and hydration, stained with haematoxylin-eosin (HE) and sealed for microscopic observation using an ASP200S dehydrator, EG1150 embedding machine, and RM2245 paraffin slicer (Leica, Germany).

\section{Determination of Superoxide Dismutase (SOD), Glutathione (GSH), Glutathione Peroxidase (GSH-Px) and Malondialdehyde (MDA) Contents in the Serum}

The contents of GSH, MDA, and SOD were determined using colorimetry, and the content of GSH-PX was determined using a nonenzymatic immunoassay. First, the GSH antibody was coated on a microporous plate to prepare a solid phase antibody, and an antibody-antigen-enzyme labelled antibody complex was formed. Finally, the colour of the tested serum and complex were converted to yellow under the action of horseradish peroxidase (HRP), 
indicating the GSH content. Using colorimetry, the amount of MDA in the degradation product of peroxidised lipids condensed with thiobarbituric acid (TBA) formed a red colour, with a maximum absorption peak at $532 \mathrm{~nm}$. SOD was detected using colorimetry, where pyrogallol was selfoxidized to produce superoxide anion free radicals, and the ability of SOD to decompose $\mathrm{O} 2$ was proportional to the activity of SOD, which was converted into the SOD content. GSH-PX was analysed using a nonenzymatic immunoassay. The purified GSH-PX antigen was coated on a microplate to form a solid phase antigen, and the detection samples formed an antibody-antigen-enzyme-labelled antibody complex. After washing, the substrate TMB was used to develop the colour, which was converted to blue by the catalysis of the HRP enzyme. The colour depth was positively correlated with the GSH-PX content. The absorbance value (OD) was measured using a plate reader at a wavelength of $450 \mathrm{~nm}$ to determine the GSH-PX concentration. The steps described above were performed strictly in accordance with the instructions of the kits (GSH-PX -20181010, GSH- 20181110, MDA -20181100, and SOD-20181111, Nanjing Jiancheng, China).

\section{Immunofluorescence Staining}

Paraffin sections were dewaxed (RM2035 pathological slicer, Shanghai Leica Instrument Co., Ltd.) and heated in EDTA (G1206, Solarbio, pH 8.0) for antigen retrieval for 8 min. The hydrogen peroxide was added, the slices were slightly dried, and a histochemical pen (WG1066, Servicebio) was used to draw a circle around the tissue (to prevent the antibody from flowing away). Sections were blocked with serum (bovine serum albumin (VI), G20191212, Solarbio) and then incubated at $4{ }^{\circ} \mathrm{C}$ overnight with one or two of the following primary antibodies diluted in PBS: ATP5A1 mouse monoclonal antibody (lot: 10002127, 1:300) or UCP2 rabbit polyclonal antibody (lot: 00069126, 1:200, Proteintech Group, Inc. (USA)). Sections were rinsed and incubated with secondary antibodies conjugated to HRP of the corresponding species for $50 \mathrm{~min}$ at room temperature. Nuclei were stained with DAPI. Tissue autofluorescence (autofluorescence quenching agent, G1221, Servicebio) was quenched. The slices were slightly dried and sealed with anti-fluorescence quenching tablets (G1401, Servicebio). The slices were placed under the microscope to capture images (XSP-PM21AY fluorescence microscopic imaging system, Shanghai Optical instrument Co., Ltd.). The nuclei stained with DAPI were blue upon UV excitation, and positive staining was red and green in sections labelled with the corresponding fluorophore.

\section{Transmission Electron Microscopy (TEM)}

The brain tissues were fixed with 4\% neutral glutaraldehyde followed by $1 \%$ osmic acid. The samples were eluted with acetone. After embedding in a porous rubber template, samples were dried in the oven to form an entrapment block. The samples were cut into $70 \mathrm{~nm}$-thick slices with a Leica ultrafine instrument (EMUC6 type, Leica, Germany). The sections were stained with uranyl acetate and lead citrate. Transmission electron microscopy (H-7650, Japan Hitachi Co., Ltd.) was used to observe the sections and capture images.

\section{Mitochondrial Extraction}

1. For the preparation of the brain tissue homogenate, 100-200 mg of brain tissue were rinsed with normal saline, blotted with filter paper, cut into pieces and placed in a small volume glass homogenizer. One millilitre of ice-cold Lysis Buffer was added to the sample in a $0{ }^{\circ} \mathrm{C}$ ice bath and the microstructure was grinded 20 times. 2. The sample was transferred to centrifuge tube and centrifuged at $1000 \times \mathrm{g}$ for $5 \mathrm{~min}$ at $4{ }^{\circ} \mathrm{C}$. 3 . The supernatant was extracted and centrifuged at $1000 \times \mathrm{g}$ for $5 \mathrm{~min}$ at $4^{\circ} \mathrm{C}$. 4 . The supernatant was centrifuged at $12,000 \times \mathrm{g}$ for $10 \mathrm{~min}$ at $4{ }^{\circ} \mathrm{C}$ before being transferred to a new centrifuge tube, and the mitochondria were precipitated at the bottom of the tube. 5 . Then, $0.5 \mathrm{~mL}$ of Wash Buffer was added to the mitochondrial precipitate to resuspend the mitochondrial precipitate, and centrifuged at $1000 \times \mathrm{g}$ for $5 \mathrm{~min}$ at $4{ }^{\circ} \mathrm{C}$. 6 . The supernatant was transferred to a new centrifuge tube and centrifuged at $12,000 \times \mathrm{g}$ for 10 min at $4{ }^{\circ} \mathrm{C}$. The supernatant was discarded, and high-purity mitochondria were precipitated at the bottom of the tube. 7 . The mitochondrial precipitate was suspended in $50-100 \mu \mathrm{L}$ of Storage Buffer and stored at $-70{ }^{\circ} \mathrm{C}$. The instructions of the mitochondrial extraction kit were strictly followed (SM0020, Beijing Solarbio Science \& Technology Co., Ltd.).

\section{Quantitative Real-Time PCR (qRT-PCR) Analysis}

Total RNA was extracted from gastrocnemius muscle tissue samples with TRIzol reagent. All qRT-PCR analyses were performed using the ABI7500 Real-Time PCR System (Applied Biosystems). The cDNA templates were generated via reverse transcription using the PrimeScript ${ }^{\mathrm{TM}}$ RT 
Reagent Kit with gDNA Eraser (TaKaRa, Japan). Real-time PCR was performed in a CFX96 instrument after reverse transcription using the following conditions: after $95^{\circ} \mathrm{C}$ for $30 \mathrm{~s}$, followed by 40 cycles of $95^{\circ} \mathrm{C}$ for $5 \mathrm{~s}$ and $60^{\circ} \mathrm{C}$ for 40 $\mathrm{s}$. The relative mRNA expression was calculated using the $2-\Delta \Delta \mathrm{Ct}$ comparative method. The primer sequences of all target genes are shown in Table 1.

\section{Western Blot Analysis}

The supernatant was collected, and the rat brain mitochondrial protein concentration was determined using the bicinchoninic acid (BCA) method. The extracted cellular proteins were subjected to SDS-PAGE, transferred to NC membranes $(0.45 \mu \mathrm{m})$, and then blocked with $3 \%$ BSA in TBST for 30 $\min$. The membranes were subsequently probed with an antiAMPK antibody (diluted 1:2000, rabbit monoclonal antibody, Abcam), anti-PPAR $\alpha$ antibody (diluted 1:1000, mouse monoclonal antibody, GeneTex), anti-PGC-1 $\alpha$ antibody (diluted 1:4000, rabbit polyclonal antibody, Abcam), anti-UCP2 antibody (diluted 1:2000, rabbit polyclonal antibody, Proteintech), or anti-p-AMPK antibody (diluted 1:5000, rabbit polyclonal antibody, Abcam) overnight at $4{ }^{\circ} \mathrm{C}$. The membrane was incubated with the secondary antibody (CWBiotech, China) for $90 \mathrm{~min}$ after the membrane had been washed with TBST 6 times for 3 min each. The exposed film was scanned directly, and ImageJ software changed the picture format from JPEG to Tif. Total Lab Quant V11.5 (Newcastle upon Tyne, UK) was used to calculate the value of the integral optical density (IOD) of the protein bands.

\section{Data Processing and Multivariate Analysis}

All data were processed using SPSS 22.0 statistical software and are presented as the means \pm standard deviations (SDs). Multiple groups were compared using one-way analysis of variance (ANOVA), assuming homogeneity of variances, and two-way ANOVA followed by LSD test were used to analyse the MWT data. If the variance was not uniform, Dunnett's T3 method was used. $\mathrm{P}<0.05$ or $\mathrm{P}<0.01$ was defined as a significant difference.

\section{Results}

\section{Bioactive Components of SMYZD}

The main active components of Panax ginseng C.A. Mey, Gastrodia elata Bl., Euonymus alatus Sieb. (Thunb.), and Ligusticum chuanxiong Hort. were detected using LC-MS. These compounds were ginsenosides, gastrodin, $\beta$ sitosterol, and ferulic acid, which are shown in Figure 1.

\section{SMYZD Improves CCH-Induced Spatial Memory and Learning Deficits}

As shown in Figure 2, the Morris water maze test was performed to determine the effect of SMYZD on CCHinduced cognitive impairment at the 8th week after intervention. The escape latencies of Model group were markedly longer (40.29 \pm 36.54 vs $17.52 \pm 21.42$ s) beginning on the 3rd day (trial days 3 and $4, P<0.05$ ) and the rats spent less time in the target quadrant and exhibited fewer platform crossings than Sham rats $(19.31 \pm 3.98$ vs $28.15 \pm 5.21 \mathrm{~s})$ $(P<0.01)(2.7 \pm 2.36$ vs $7.3 \pm 3.2 \mathrm{~s})(P<0.05)$. After treatment with SMYZD for 8 weeks, the escape latency of the SMYZ-H group was improved $(18.36 \pm 22.4248 .02$ $\pm 36.59 \mathrm{~s}$ ), the SMYZ-H group of rats spent a markedly longer time in the target quadrant $(25.44 \pm 7.31$ vs $19.31 \pm$ 3.98 s) $(P<0.01)$, and crossed the platform location more times $(7.1 \pm 4.12$ vs $2.7 \pm 2.36 \mathrm{~s})(P<0.05)$ than the Model group, suggesting that SMYZD may partially ameliorate the spatial memory and learning impairments caused by $\mathrm{CCH}$.

\section{SMYZD Reduces Chronic Ischemic Changes in the Rat Cerebral Cortex Caused by $\mathrm{VCl}$}

With the use of light microscope, pyramidal cells, granulosa cells and neurons in the cerebral cortex of the sham operation group were normally arranged and closely connected, and no inflammatory cells or chronic ischemic changes were observed. The pyramidal cells, granulosa cells and neurons in the model group were sparse and scattered, the number of cells decreased significantly,

Table I Primers Used for Real-Time Quantitative PCR

\begin{tabular}{|l|l|l|}
\hline Gene & Forward Primer (5'-3') & Reverse Primer (5'-3') \\
\hline AMPK & GACCTCGGTCAAGTGTCGATTC & AACGGGCTAAAGCAGTGATAAGA \\
PPAR $\alpha$ & GGGACAAGGCCTCAGGATACCACTA & GACATCCCGACGGACAGGCACT \\
PGC- $1 \alpha$ & CAAGGTCCCCAGGCAGTAGAT & CTTTCGTGCTCATTGGCTTCATA \\
UCP2 & CCCAATGTTGCCCGAAAT & CCCGAAGGCAGAAGTGAAG \\
ATP5A & TGCTTTCTTGTCTCATGTTGTG & GTTTGTTACGATTTCCTTCAGC \\
\hline
\end{tabular}




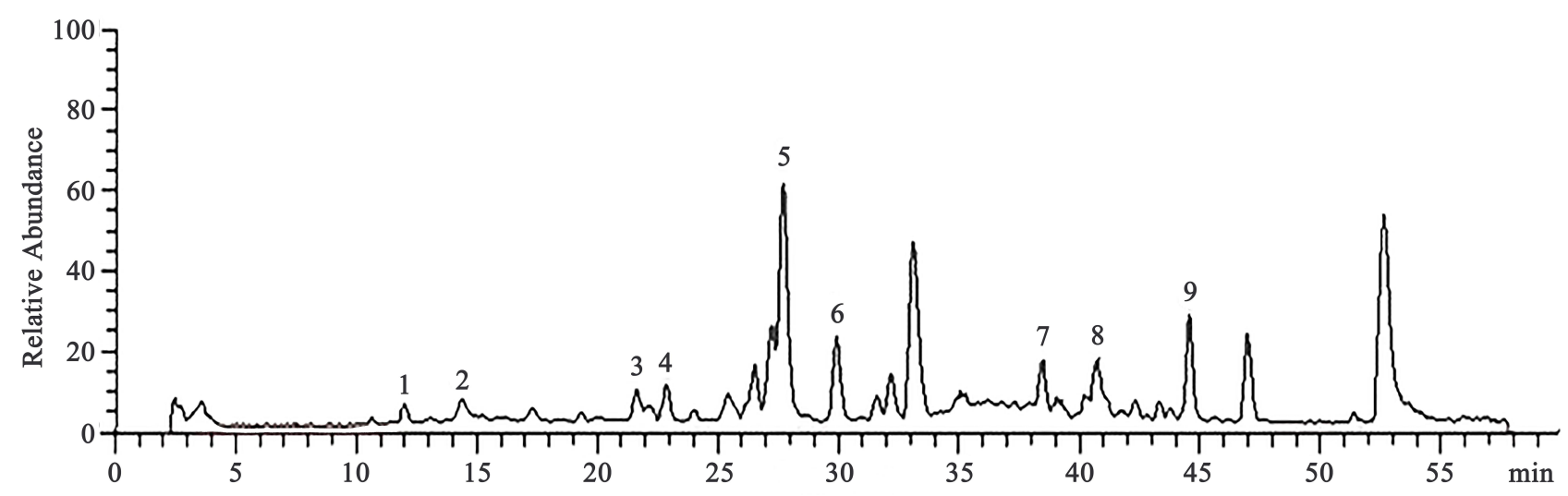

Figure I LC-MS chromatogram of water extract of SMYZD I. Alatamine; 2. $\beta$-sitosterol; 3. FerulicAcid; 4. ProtocatechuicAcid; 5. Gastrodin; 6. 4-Hydroxybenzyl alcohol; 7. GinsenosideRgI; 8. Ginsenoside Re; 9. Ginsenoside Rb3.

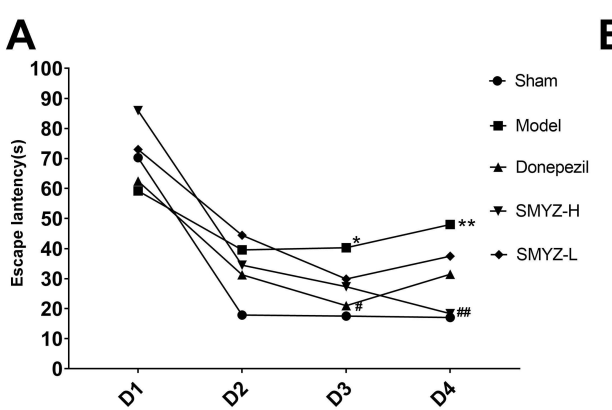

D Place navigation(Learning)

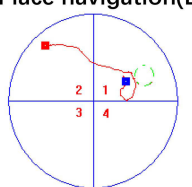

Probe test(Memory)

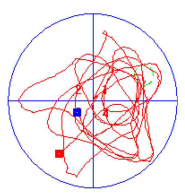

Sham
B

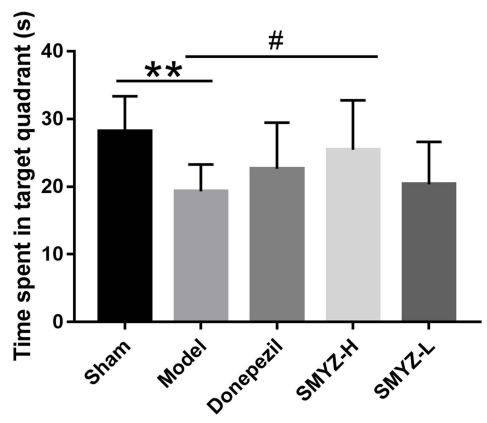

C

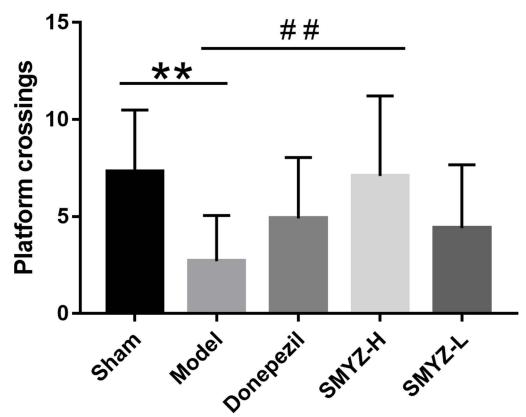

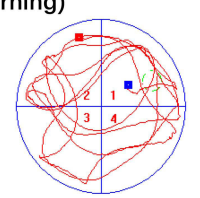
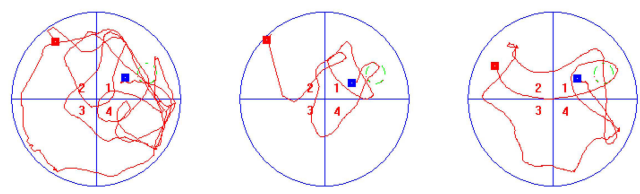

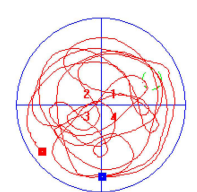

Model

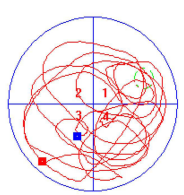

Donepezil

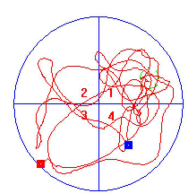

SMYZ-H

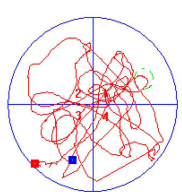

SMYZ-L

Figure 2 The Morris water maze was used to assess spatial memory and learning abilities. Escape latency (A) was the mean value of each platform trial for 4 consecutive days, and the time the rats spent in the target quadrant (B) and number of platform crossings (C) were measured during the probe trials. (D) Typical swimming paths of rats in the sham, model, donepezil, SMYZ-H, and SMYZ-L groups in the MWM during learning (top panel) and memory probe tests (bottom panel). *P < 0.05 compared with the sham group; ${ }^{\#} P<0.05$ compared with the model group. ${ }^{* * P}<0.01$ compared with the sham group; ${ }^{\#} P<0.01$ compared with the model group.

inflammatory cells and chronic ischemic changes can be observed. Compared with model group, the number of cells increased significantly, the cell structure was more complete and the arrangement of the above cells was better in three drug group. Thus, SMYZD improved the pathological changes in the morphology of the cerebral cortex in rats with VCI caused by bilateral common carotid artery ligation, as shown in Figure 3.

\section{Effects of SMYZD on GSH, GSH-Px,} MDA and SOD Contents in the Serum of Rats with $\mathrm{VCl}$

Compared with the sham operation group, the serum GSH and GSH-PX concentrations in the model group decreased significantly, while the MDA content increased significantly $(P<0.05, P<0.01)$. Compared with the model 


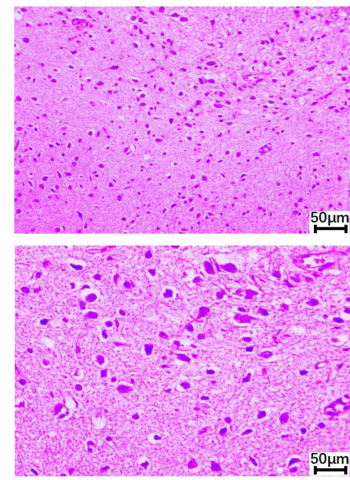

Sham

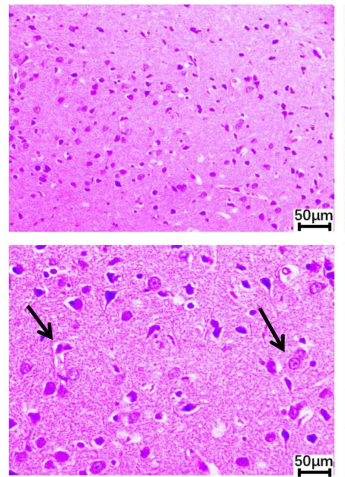

Model

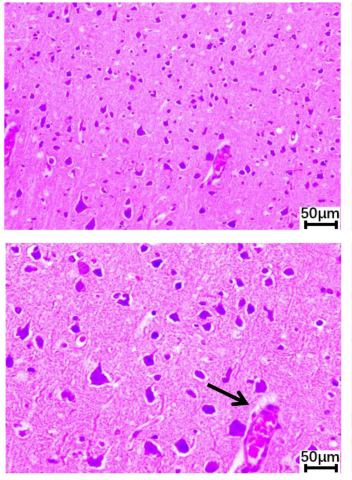

Donepezil

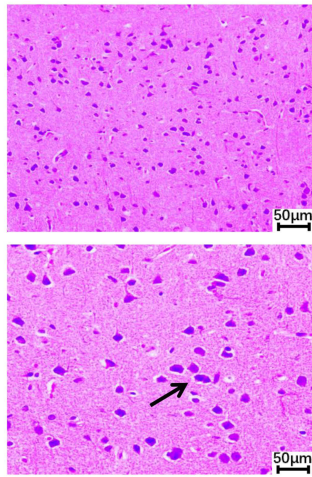

SMYZ-H

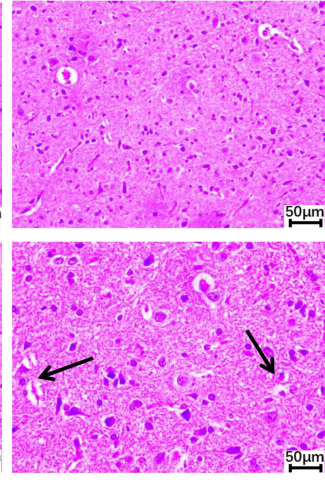

SMYZ-L

Figure 3 Effect on the pathomorphology of the cerebral cortex from rats assessed using HE staining at 8 weeks after the SMYZD intervention. Inflammatory cells and chronic ischaemic changes are labelled with black arrows $($ bar $=50 \mu \mathrm{m})$.

group, the GSH and GSH-PX contents in the donepezil hydrochloride group and SMYZD high-dose group were significantly increased, the MDA content was significantly decreased $(P<0.05)$, and the SOD content in the SMYZD high-dose group was significantly increased $(P<0.05, P<$ 0.01). Based on these results, SMYZD alleviates oxidative stress-induced injury to the mitochondria of VCI rats (Figure 4).

\section{SMYZD Increases the Number of Mitochondria in Rats with $\mathrm{VCl}$}

ATP5A, a marker of mitochondria, served as a negative control in the sham group. Fewer mitochondria were observed in the model group. After the intervention, the fluorescence images showed that the number of mitochondria increased to a certain extent, indicating that SMYZD increased the number of mitochondria in rats with VCI caused by bilateral common carotid artery ligation, as shown in Figure 5.

\section{Observation of the Mitochondrial}

\section{Structure in the Brains of Rats with $\mathrm{VCl}$}

Transmission electron microscopy (TEM) was used to observe the tissues from rats in each group. The mitochondrial structure of brain tissue of the sham operation group was basically normal and neatly arranged, the membrane shape was basically intact, the mitochondrial cristae were dense, and no obvious swelling or vacuole formation was detected. In the model group, the structure of mitochondria was obviously changed, the membrane of the mitochondria was blurred, part of the membrane was ruptured, and the mitochondrial cristae were broken and loosely dissolved, accompanied by a reduction or disappearance of matrix particles. The disturbance of mitochondrial structure in drug groups was significantly improved, the mitochondrial membrane was generally clear, and the number of mitochondrial cristae increased. The results are shown in Figure 6.

\section{Measurement of AMPK, PPAR $\alpha$, PGC-I $\alpha$, UCP2, and ATP5A mRNA Expression Levels by RT-PCR}

Mitochondria are the target organelles of oxidative stressinduced injury, and mitochondrial dysfunctional leads to less ATP production. Activated AMPK, PPAR $\alpha$, and PGC$1 \alpha$ are important regulators of mitochondrial biogenesis. UCP2 has a crucial role in the regulation of oxidative stress, and ATP5A is the core hub controlling the structure and function of mitochondria. Real-time PCR was used to evaluate the mRNA expression levels of AMPK, PPAR $\alpha$, PGC-1 $\alpha$, UCP2 and ATP5A in the brain mitochondrial samples from each group to further verify this phenomenon. The AMPK, PPAR $\alpha$, PGC- $1 \alpha$, and ATP5A mRNA expression levels in the model group were significantly lower than in the sham group $(P<0.05, P<0.01)$. After the SMYZD intervention, AMPK, PPAR $\alpha$, PGC- $1 \alpha$, and ATP5A mRNA expression was significantly increased compared with the model group $(P<0.05, P<0.01)$. Compared with the sham group, the UCP2 mRNA was expressed at significantly higher levels in the model group $(P<0.01)$. Compared with the model group, the UCP2 mRNA was expressed at significantly lower levels in the donepezil, SMYZ-H and SMYZ-L groups $(P<0.05, P<$ 

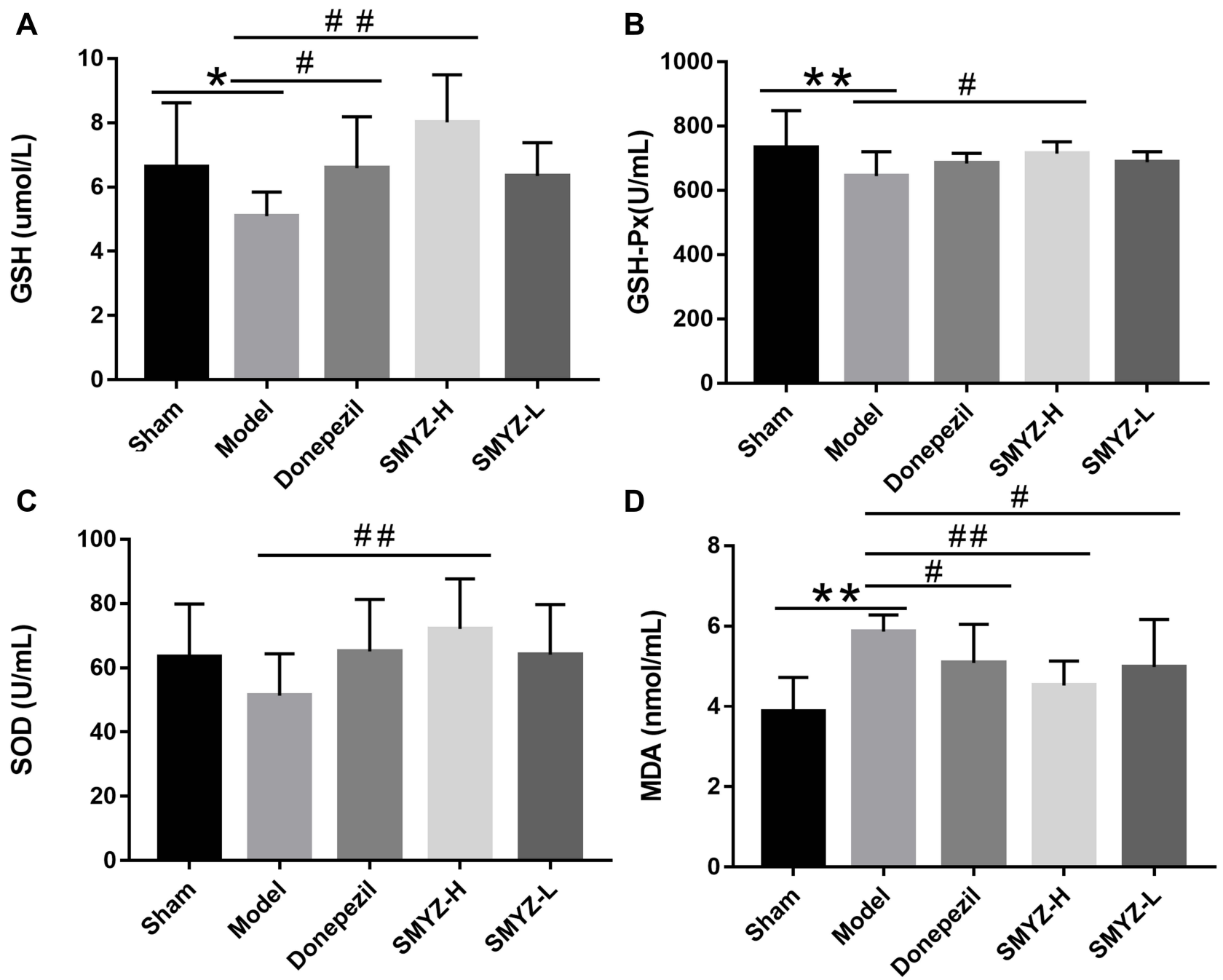

Figure 4 GSH (A), GSH-Px (B), SOD (C) and MDA (D) contents in the serum from rats with $\mathrm{VCl}$ in each group. $* P<0.05$ compared with the sham group; ${ }^{\#} P<0.05$ compared with the model group. $* * P<0.01$ compared with the sham group; ${ }^{\# \#} P<0.01$ compared with the model group. ( $\mathrm{n}=10$ ).

0.01). Therefore, SMYZD promotes mitochondrial energy metabolism in VCI rats via the AMPK, PPAR $\alpha, \mathrm{PGC}-1 \alpha$, and UCP2 pathways (Figure 7).

\section{Measurement of AMPK, PAMPK, PPAR $\alpha$, PGC-I $\alpha$, UCP2, and ATP5A Protein \\ Levels Using Western Blotting}

We detected the expression of the indicators listed above at the mRNA level. Next, Western blotting was used to further verify their expression at the protein level. The levels of the pAMPK (B), PPAR $\alpha$ (C), PGC-1 $\alpha$ (D), UCP2 (E), and ATP5A (F) proteins were decreased in the model group compared with the sham group, and the differences were significant. After the SMYZD treatment, levels of the pAMPK, PGC-1 $\alpha$, UCP2, and ATP5A proteins were increased significantly compared with the model group $(\mathrm{P}<0.05)$. The differences in AMPK protein expression levels were not significant, suggesting that AMPK plays a role in this pathway upon its phosphorylation (Figure 8).

\section{Discussion}

In this study, we revealed that SMYZD improves mitochondrial structure and energy metabolism to ameliorate chronic cerebral hypoperfusion, which can induce vascular cognitive impairment. First, decreased expression of pAMPK, PPAR $\alpha$, PGC- $1 \alpha$, and UCP2 in the mitochondria of the brains of VCI rats was detected, accompanied by pathological changes in the mitochondria. More importantly, we verified that the therapeutic effect of SMYZD is achieved by activating the AMPK/PPAR $\alpha / \mathrm{PGC}-1 \alpha /$ 

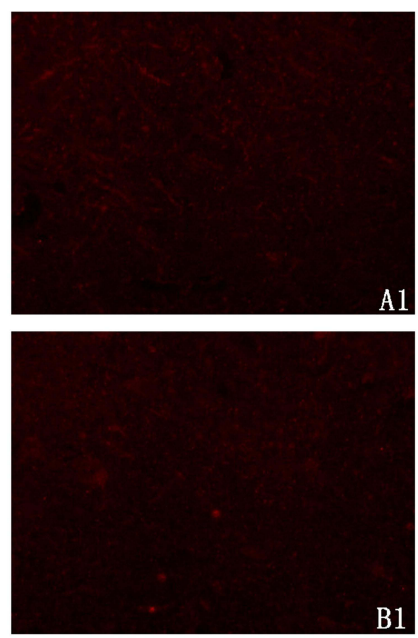

B1
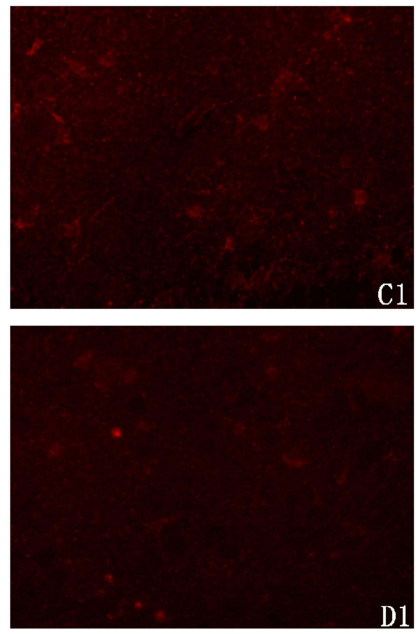

D1

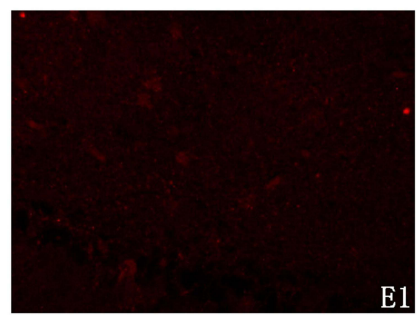

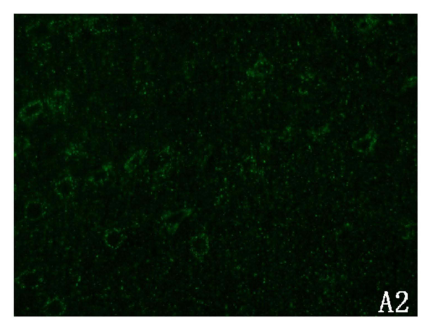
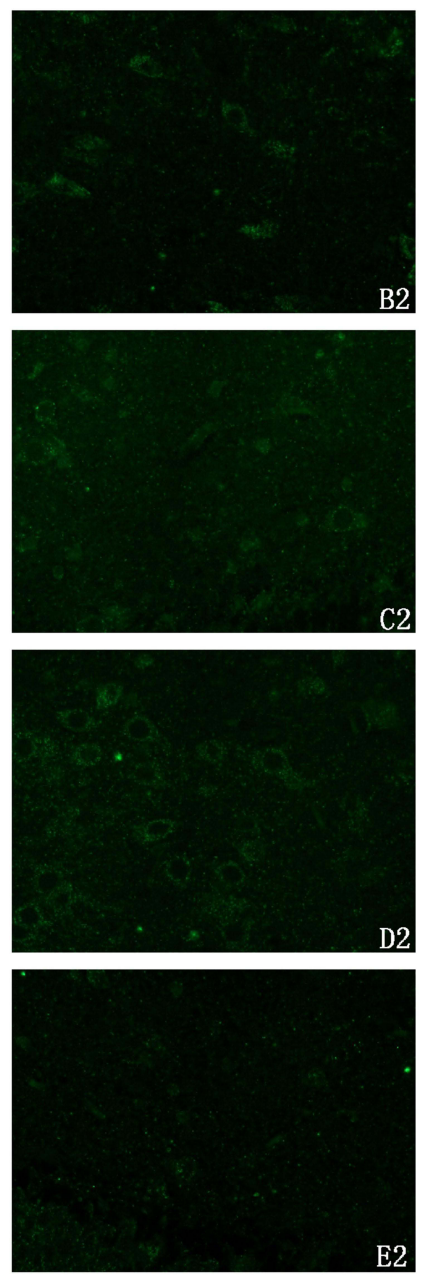
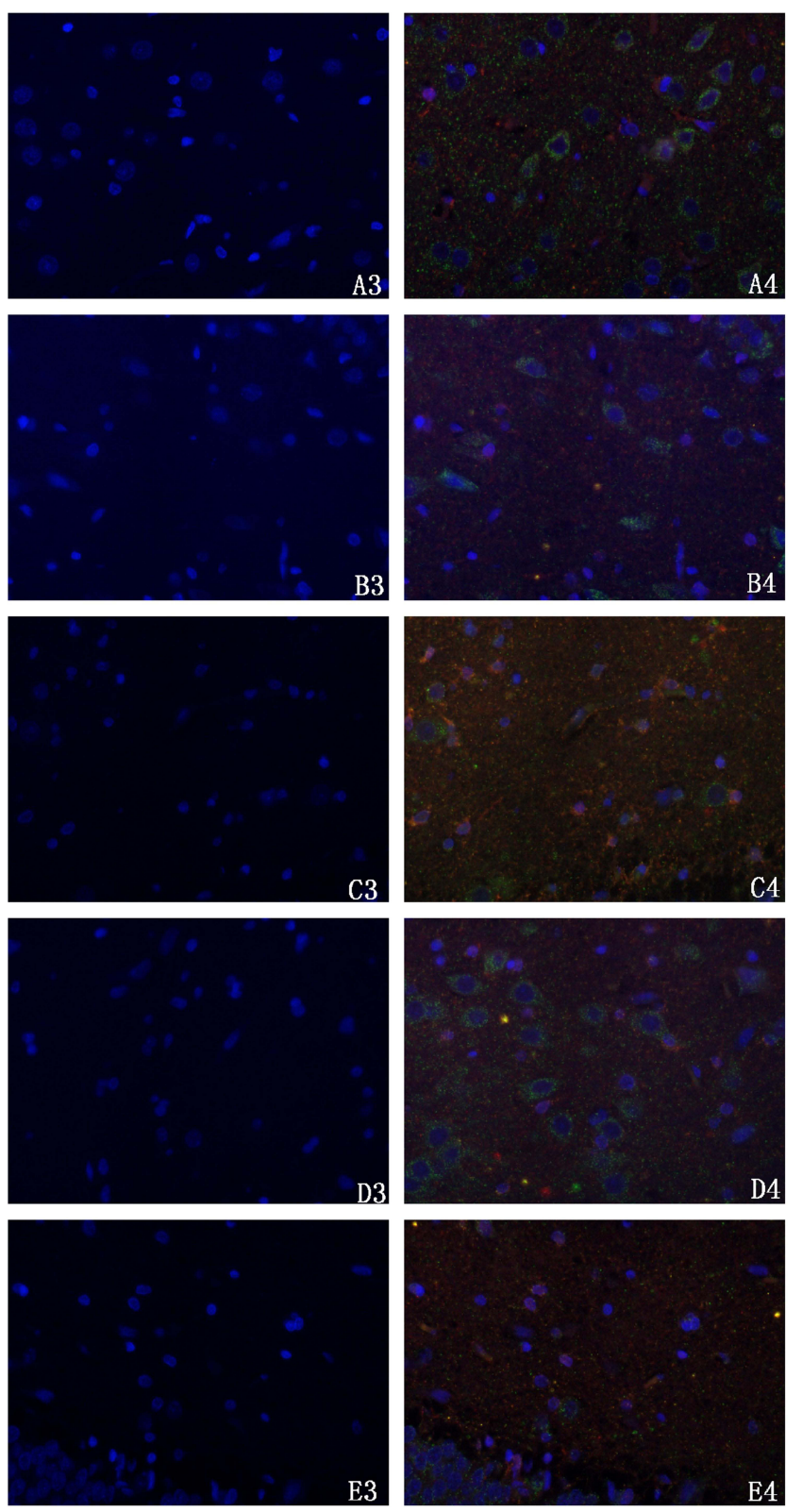

Figure 5 Effect of SMYZD on mitochondrial uncoupling protein $2(\mathrm{UCP} 2)$ expression in the cerebral cortex of rats with chronic cerebral ischaemia $(\times 400)$.

Notes: Red fluorescence represents mitochondrial UCP2, green fluorescence represents the expression of the mitochondrial syncytial protease ATP5A, a mitochondrial marker; its expression was localized to the mitochondrial inner membrane or peripheral membrane. Blue fluorescence represents the nucleus. A:Sham; B:Model; C: Donepezil; D:SMYZ-H;E:SMYZ-L.

UCP2 signalling pathway to improve mitochondrial structure and alleviate pathological injury and oxidative stressinduced injury in the brains of VCI rats.

SMYZD contains a variety of active substances. These compounds include gastrodin, ferulic acid, ginsenosides, and $\beta$-sitosterol. Gastrodin (4-(hydroxymethyl) phenyl- $\beta$ D-glucopyranoside) is a phenolic glycoside obtained from Chinese herbal medicine (Gastrodia elata Bl.). It possesses antioxidant, anti-inflammatory and antiapoptotic properties and is widely used to treat nervous system diseases and cardiovascular and cerebrovascular diseases. GAS crosses the blood-brain barrier, thus reducing the effects of different stressors on the cognition of experimental animals. ${ }^{29}$ GAS has been shown to regulate mitochondrial dynamics and maintain the structure and function of mitochondria to protect cells from oxidative damage. ${ }^{30}$ Ferulic acid reduces oxidative stress and prevents cell apoptosis by activating PPAR $\gamma .{ }^{31}$ Furthermore, ferulic acid reduces the oxidative stress associated with vascular injury and endothelial cells in mice and improves mitochondrial 


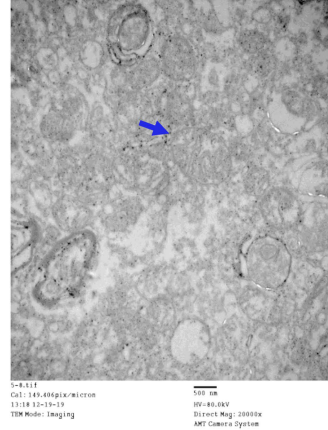

Sham

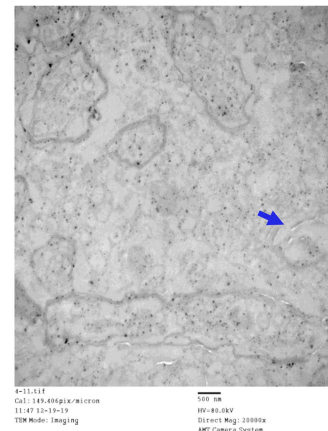

Model

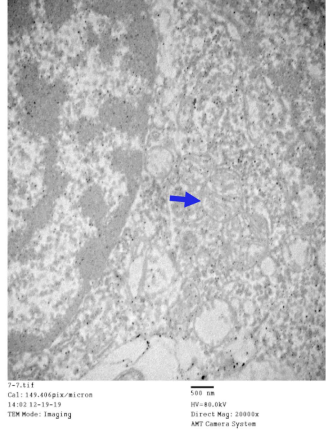

Donepezil

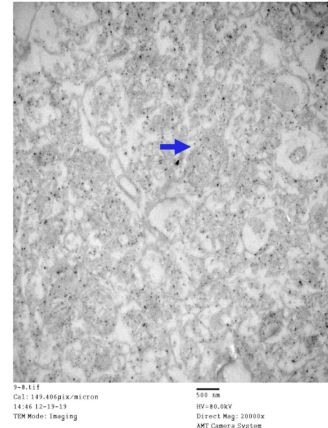

SMYZ-H

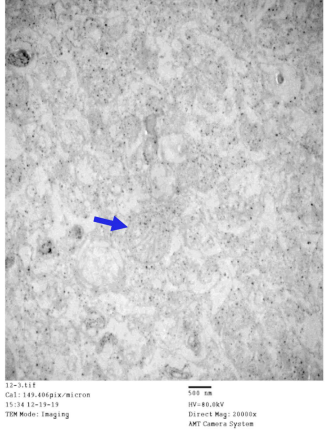

SMYZ-L

Figure 6 Effect of SMYZD on the mitochondrial subcellular structure in the brain tissue of $\mathrm{VCl}$ rats $(\times 20,000)$. TEM observations of the mitochondrial structures in rats from each group. Mitochondria are labelled with blue arrows $($ bar $=500 \mathrm{~nm})$.

biogenesis. ${ }^{32}$ Ginseng has been used to treat various diseases and has proven its great efficacy in the control of central nervous system diseases. These effects are exerted through its neurogenesis, antiapoptosis and antioxidant properties, and inhibition of mitochondrial dysfunction. ${ }^{33}$ The major ginsenosides Rb1, Rg1, Rb2, Rc, Rd, and Re dominate the chemical profile of this genus in vivo and in vitro. ${ }^{34} \mathrm{Rb} 1$ or $\mathrm{Rg} 1$ increases the activity of complexes I, III and V, increases the level of ATP, and improves the function of mitochondria. ${ }^{35}$ Two major ginsenosides may affect the kinetics of mitochondrial renewal. ${ }^{36}$ It significantly improves the redox state of cells, improves the function of the glutathione system, and increases SOD activity to protect the mitochondria, indicating that ginsenosides $\mathrm{Rb} 1$ and $\mathrm{Rg} 1$ have neuroprotective potential. ${ }^{37} \mathrm{In}$ addition, ginsenoside $\mathrm{Rb} 1$ regulates mitochondrial energy metabolism. ${ }^{38} \beta$-Sitosterol is a bioactive compound that naturally exists in the plant cell membrane and has a variety of biological functions, such as immune regulation, anti-inflammatory, and antioxidant activities. ${ }^{39} \beta$ -
A

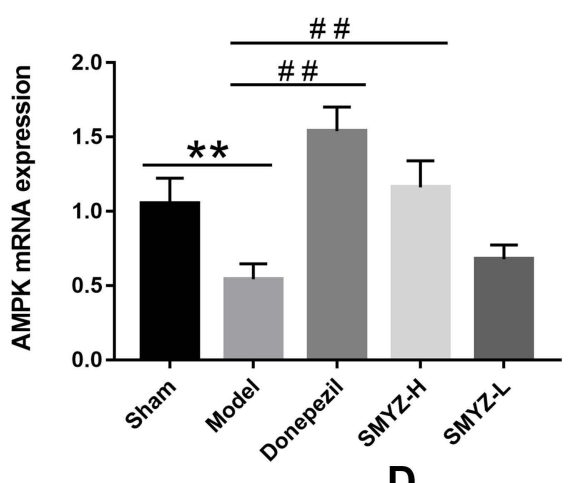

B

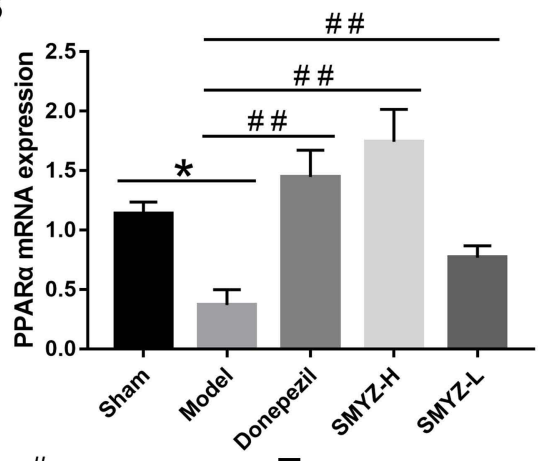

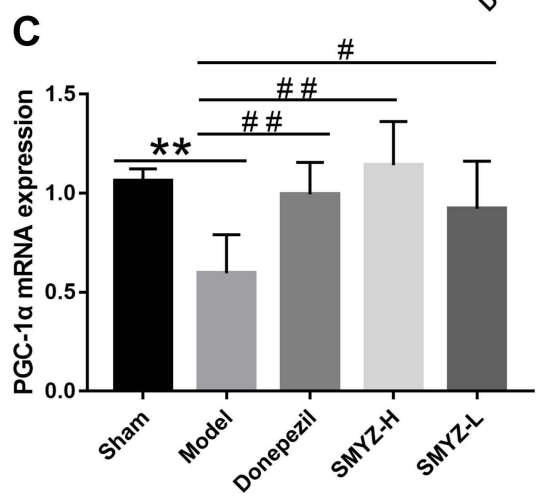
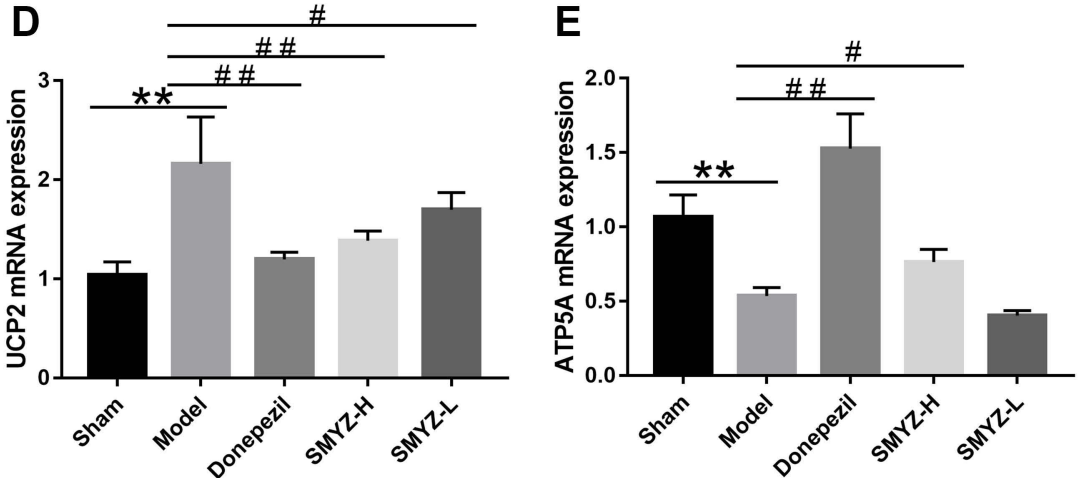

Figure 7 AMPK (A), PPAR $\alpha$ (B), PGC-I $\alpha$ (C), UCP2 (D), and ATP5A (E) mRNA expression in each group. *P $<0.05$ compared with the sham group; ${ }^{\#} P<0.05$ compared with the model group. ${ }^{*} P<<0.01$ compared with the sham group; ${ }^{\#} P<0.01$ compared with the model group. $(n=5)$. 


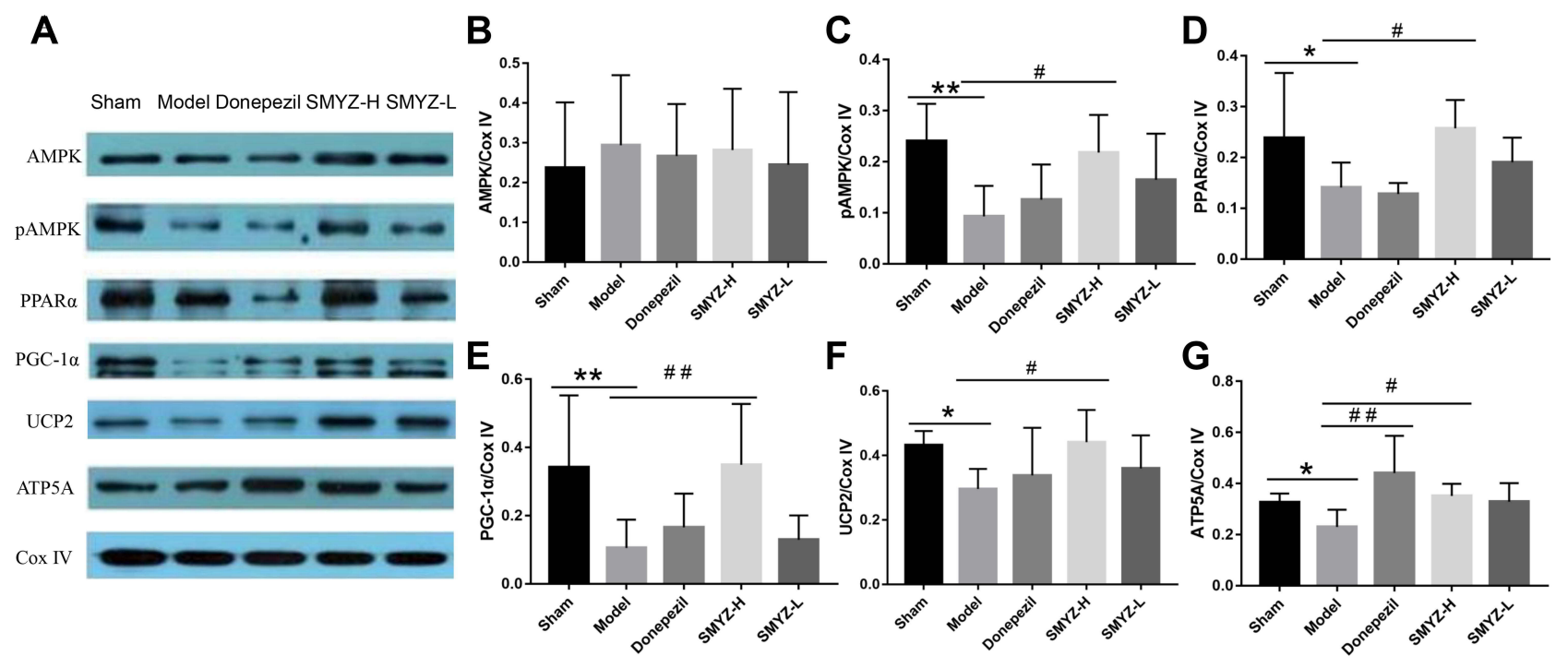

Figure 8 WB protein expression bands in each group (A) Comparison of AMPK (B), pAMPK (C), PPAR $\alpha$ (D), PGC-I $\alpha$ (E), UCP2 (F), and ATP5A (G) protein expression in

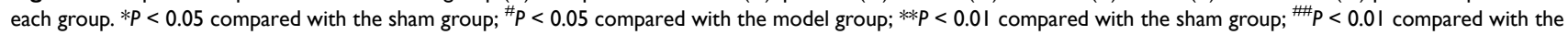
model group. $(n=5)$.

Sitosterol protects the rat heart from oxidative damage by enhancing the mitochondrial glutathione redox cycle. ${ }^{40} \beta$ Sitosterol increases the fluidity of the mitochondrial inner membrane, thus enhancing the function of mitochondria. ${ }^{41}$

Based on the aforementioned studies examining the pharmacological effects of various components of SMTZD, we infer that this treatment may restore mitochondrial structure and improve mitochondrial function by inhibiting oxidative stress and exerts a synergistic effect on improving the cognition of VCI rats.

Oxidative stress is usually defined as an imbalance between oxidants and antioxidants at the cellular or individual level. Oxidative damage is one of the results of this imbalance, including apoptosis and tissue damage, and eventually leads to various diseases. ${ }^{42}$ Oxidative stress is the main mechanism of BCCAO-induced brain injury in the early stage, which leads to vascular endothelial cell injury, intracellular calcium overload, mitochondrial dysfunction and other consequences. Oxidative stress-induced mitochondrial dysfunction in the hippocampus is considered to play a major role in the pathogenesis of VD. ${ }^{43}$ The enzymes involved in antioxidant defences include superoxide dismutase (SOD) and glutathione peroxidase, as well as water- and fat-soluble antioxidants, such as glutathione. ${ }^{44}$ MDA is a product of lipid peroxidation and a recognized indicator of oxidative stress. It is a toxic molecule that destroys the structure of the cell membrane. ${ }^{45}$ The contents of SOD and MDA reflect the level of oxidative stress in brain tissue. The level of GSH is closely related to the steady state of the redox system. Monitoring the change in GSH levels is helpful to evaluate the effect of treatment. ${ }^{46}$ GSH-PX directly or indirectly functions as an antioxidant in the body. ${ }^{47}$ At the same time, as an indicator of oxidative stress, its content represents the strength of the antioxidant capacity of the body. Central nervous system injury is usually accompanied by damage to nerve tissue and cells and a reduction in protein levels in cells, cerebrospinal fluid and blood. In the present study, the serum MDA level increased and the levels of GSH, GSH-PX, and SOD decreased in VCI rats. Consistent with previous pharmacological studies, SMYZD reduced the level of MDA and increased the activity of GSH, GSH-PX, and SOD in VCI rats, suggesting that it inhibits oxidative stress in VCI rats.

As the main regulator of energy homeostasis, AMPK regulates more aspects and functions of mitochondria. AMPK regulates at least three basic aspects of mitochondrial homeostasis: biogenesis, fission and mitochondrial phagocytosis. Almost all types of mitochondrial damage or defects activate AMPK, and thus AMPK acts as the guardian of mitochondria. ${ }^{48}$ AMPK binds to downstream effectors to ensure optimal mitochondrial function and contributes to the regulation of mitochondrial biogenesis. The AMPK/PPAR $\alpha$ pathway regulates the content and function of mitochondria. ${ }^{49}$ The AMPK/ PGC-1 $\alpha$ pathway not only enhances mitochondrial 
biogenesis ${ }^{50}$ but also participates in the regulation of mitochondrial energy metabolism. ${ }^{51}$ PGC-1 $\alpha$ (peroxisome proliferator-activated receptor gamma coactivator 1-alpha) is a transcriptional coactivator of many genes involved in energy management and mitochondrial biogenesis. $^{52}$ AMPK regulates the activity of UCP2 by increasing the expression of PGC- $1 \alpha$, and the upstream region of $\mathrm{PGC}-1 \alpha$ is regulated by AMPK. ${ }^{53}$ AMPK activates PPAR $\alpha$ to upregulate $\mathrm{UCP} 2$, indicating that the upstream region of PPAR $\alpha$ may be regulated by AMPK. ${ }^{54}$ In addition, the expression levels of AMPK, pAMPK, PPAR $\alpha$, PGC-1 $\alpha$, and UCP2 in the brain mitochondria of VCI rats were decreased. The expression of UCP2 in the real-time PCR experiment was opposite to the Western blot analysis. The potential explanations are provided below. Mitochondria are not only the main site of energy production but also the main organelles involved in ischaemia. Mitochondria extracted from ischaemic and hypoxic myocardium show a decreased oxidation ability, and ischaemia resulted in decreased oxygen uptake, slowed ATP consumption, decreased ATP synthesis and increased expression of UCP $2,{ }^{55}$ indicating that ischaemia and hypoxia induced by BCCAO lead to abnormalities in mitochondrial respiratory function and oxidative phosphorylation. Moreover, the expression of the UCP2 mRNA is regulated by many factors in vivo, such as miRNAs ${ }^{56}$ and growth hormones. $^{57}$ In addition, genetic polymorphisms in UCP2 have been reported, which may lead to changes in the structure/function/activity of gene products. ${ }^{58}$

At the same time, SMYZD reversed the expression level of the aforementioned indexes. Therefore, we propose that SMYZD may improve the structure of mitochondria by activating the AMPK, PPAR $\alpha$, PGC- $1 \alpha$ and UCP2 signaling pathway and then promote mitochondrial biogenesis, to ameliorate the cognitive impairment observed in VCI rats.

\section{Conclusions}

Surprisingly, we found that SMYZD not only improved oxidative stress-induced injury but also ameliorated damage to the mitochondrial structure through the AMPK/PPAR $\alpha / \mathrm{PGC}-1 \alpha / \mathrm{UCP} 2$ signaling pathway, therefore improve the learning and memory abilities of rats with VCI. These results may provide insights into the novel mechanisms associated with the positive effects of SMYZD and further verify its therapeutic effect on VD.

\section{Funding}

This journal is supported by the G20 Engineering innovation research project of Beijing Municipal Science and Technology Plan (No.Z171100001717016). Also is supported by the National Science and Technology Major Project for "Essential new drug research and development" (No.2019ZX09301114). We are also grateful to Professor Wei Yun of Beijing University of Chemical Technology for her help in pharmacy.

\section{Disclosure}

The authors report no conflicts of interest in this work.

\section{References}

1. Liu DD, Yuan X, Chu SF, et al. CZ-7, a new derivative of Claulansine F, ameliorates 2VO-induced vascular dementia in rats through a Nrf2-mediated antioxidant responses. Acta Pharmacol Sin. 2019;40(4):425-440. doi:10.1038/s41401-018-0078-7

2. Dichgans M, Leys D. Vascular cognitive impairment. Circ Res. 2017;120(3):573-591. doi:10.1161/circresaha.116.308426

3. Kalaria RN, Maestre GE, Arizaga R, et al. Alzheimer's disease and vascular dementia in developing countries: prevalence, management, and risk factors. Lancet Neurol. 2008;7(9):812-826. doi:10.1016/ s1474-4422(08)70169-8

4. Damodaran T, Müller CP, Hassan Z. Chronic cerebral hypoperfusion-induced memory impairment and hippocampal long-term potentiation deficits are improved by cholinergic stimulation in rats. Pharmacol Rep. 2019;71(3):443-448. doi:10.1016/j.pharep.2019.01.012

5. Jiang P, Chen L, Sun J, et al. Chotosan ameliorates cognitive impairment and hippocampus neuronal loss in experimental vascular dementia via activating the Nrf2-mediated antioxidant pathway. J Pharmacol Sci. 2019;139(2):105-111. doi:10.1016/j.jphs.2018.12.003

6. Sun MK. Potential therapeutics for vascular cognitive impairment and dementia. Curr Neuropharmacol. 2018;16(7):1036-1044. doi:10.2174/1570159x15666171016164734

7. Kalaria RN. The pathology and pathophysiology of vascular dementia. Neuropharmacology. 2018;134(Pt $\quad$ B):226-239. doi:10.1016/j.neuropharm.2017.12.030

8. Hase Y, Ding R, Harrison G, et al. White matter capillaries in vascular and neurodegenerative dementias. Acta Neuropathol Commun. 2019;7(1):16. doi:10.1186/s40478-019-0666-x

9. Okamoto K, Tanaka M, Kondo S. Treatment of vascular dementia. Ann N Y Acad Sci. 2002;977:507-512. doi:10.1111/j.1749-6632.2002. tb04858.x

10. Lv J, Bhatia M, Wang X. Roles of mitochondrial DNA in energy metabolism. Adv Exp Med Biol. 2017;1038:71-83. doi:10.1007/978981-10-6674-0_6

11. Magistretti PJ, Allaman I. A cellular perspective on brain energy metabolism and functional imaging. Neuron. 2015;86(4):883-901. doi:10.1016/j.neuron.2015.03.035

12. Marinangeli C, Didier S, Ahmed T, et al. AMP-activated protein kinase is essential for the maintenance of energy levels during synaptic activation. iScience. 2018;9:1-13. doi:10.1016/j.isci.2018.10.006

13. Lin SC, Hardie DG. AMPK: sensing glucose as well as cellular energy status. Cell Metab. 2018;27(2):299-313. doi:10.1016/j. cmet.2017.10.009 
14. Cao K, Zheng A, Xu J, et al. AMPK activation prevents prenatal stress-induced cognitive impairment: modulation of mitochondrial content and oxidative stress. Free Radic Biol Med. 2014;75:156-166. doi:10.1016/j.freeradbiomed.2014.07.029

15. Li S, Yang B, Du Y, et al. Targeting PPAR $\alpha$ for the treatment and understanding of cardiovascular diseases. Cell Physiol Biochem. 2018;51(6):2760-2775. doi:10.1159/000495969

16. Pierrot N, Ris L, Stancu IC, et al. Sex-regulated gene dosage effect of PPAR $\alpha$ on synaptic plasticity. Life Sci Alliance. 2019;2(2): e201800262. doi:10.26508/1sa.201800262

17. Li L, Pan R, Li R, et al. Mitochondrial biogenesis and peroxisome proliferator-activated receptor- $\gamma$ coactivator- $1 \alpha$ (PGC- $1 \alpha)$ deacetylation by physical activity: intact adipocytokine signaling is required. Diabetes. 2011;60(1):157-167. doi:10.2337/db10-0331

18. Ding Y, Zheng Y, Huang J, et al. UCP2 ameliorates mitochondrial dysfunction, inflammation, and oxidative stress in lipopolysaccharide-induced acute kidney injury. Int Immunopharmacol. 2019;71:336-349. doi:10.1016/j. intimp.2019.03.043

19. Han B, Jiang W, Liu H, et al. Upregulation of neuronal PGC-1 $\alpha$ ameliorates cognitive impairment induced by chronic cerebral hypoperfusion. Theranostics. 2020;10(6):2832-2848. doi:10.7150/ thno.37119

20. Davies KM, Strauss M, Daum B, et al. Macromolecular organization of ATP synthase and complex I in whole mitochondria. Proc Natl Acad Sci U S A. 2011;108(34):14121-14126. doi:10.1073/ pnas. 1103621108

21. Attele AS, Wu JA, Yuan CS. Ginseng pharmacology: multiple constituents and multiple actions. Biochem Pharmacol. 1999;58 (11):1685-1693. doi:10.1016/s0006-2952(99)00212-9

22. Ru W, Wang D, Xu Y, et al. Chemical constituents and bioactivities of Panax ginseng (C. A. Mey.). Drug Discov Ther. 2015;9(1):23-32. doi:10.5582/ddt.2015.01004

23. Liu SJ, Liu XY, Li JH, et al. Gastrodin attenuates microglia activation through renin-angiotensin system and Sirtuin3 pathway. Neurochem Int. 2018;120:49-63. doi:10.1016/j.neuint.2018.07.012

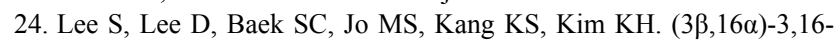
Dihydroxypregn-5-en-20-one from the Twigs of Euonymus alatus (Thunb.) Sieb. Exerts anti-inflammatory effects in LPS-stimulated RAW-264.7 macrophages. Molecules (Basel, Switzerland). 2019;24 (21):3848. doi:10.3390/molecules24213848

25. Ran X, Ma L, Peng C, Zhang H, Qin LP. Ligusticum chuanxiong Hort: a review of chemistry and pharmacology. Pharm Biol. 2011;49 (11):1180-1189. doi:10.3109/13880209.2011.576346

26. Zhang H, Cao Y, Pei H, et al. Shenmayizhi formula combined with Ginkgo extract tablets for the treatment of vascular dementia: a Randomized, Double-Blind, Controlled Trial. Evid Based Complement Alternat Med. 2020;2020:8312347. doi:10.1155/2020/8312347

27. Wu Q, Wei Y, Liu J, et al. Shenma Yizhi recipe treated mild and moderate vascular dementia patients with Qi deficiency and blood stasis induced Gan-yang hyperactivity. Chin J Integr Trad West. 2020;40(5):554-559.

28. Wu Q, Cao Y, Liu M. Traditional Chinese Medicine Shenmayizhi decoction ameliorates memory and cognitive impairment induced by scopolamine via preventing hippocampal cholinergic dysfunction in rats. Neuropsychiatr Dis Treat. 2019;15:3167-3176. doi:10.2147/ndt. S214976

29. de Oliveira MR, Brasil FB, Fürstenau CR. Evaluation of the mitochondria-related redox and bioenergetics effects of gastrodin in SH-SY5Y cells exposed to hydrogen peroxide. $J$ Mol Neurosci. 2018;64(2):242-251. doi:10.1007/s12031-018-1027-0

30. Cheng QQ, Wan YW, Yang WM, et al. Gastrodin protects H9c2 cardiomyocytes against oxidative injury by ameliorating imbalanced mitochondrial dynamics and mitochondrial dysfunction. Acta Pharmacol Sin. 2020;41(10):1314-1327. doi:10.1038/s41401-020-0382-x
31. Mahmoud AM, Hussein OE, Hozayen WG, Bin-Jumah M, Abd ElTwab SM. Ferulic acid prevents oxidative stress, inflammation, and liver injury via upregulation of $\mathrm{Nrf2} / \mathrm{HO}-1$ signaling in methotrexate-induced rats. Environ Sci Pollut Res Int. 2020;27 (8):7910-7921. doi:10.1007/s11356-019-07532-6

32. Perez-Ternero C, Werner CM, Nickel AG, et al. Ferulic acid, a bioactive component of rice bran, improves oxidative stress and mitochondrial biogenesis and dynamics in mice and in human mononuclear cells. J Nutr Biochem. 2017;48:51-61. doi:10.1016/j. jnutbio.2017.06.011

33. Rajabian A, Rameshrad M, Hosseinzadeh H. Therapeutic potential of Panax ginseng and its constituents, ginsenosides and gintonin, in neurological and neurodegenerative disorders: a patent review. Expert Opin Ther Pat. 2019;29(1):55-72. doi:10.1080/ 13543776.2019.1556258

34. Biswas T, Mathur AK, Mathur A. A literature update elucidating production of Panax ginsenosides with a special focus on strategies enriching the anti-neoplastic minor ginsenosides in ginseng preparations. Appl Microbiol Biotechnol. 2017;101(10):4009-4032. doi:10.1007/s00253-017-8279-4

35. Xu M, Ma Q, Fan C, Chen X, Zhang H, Tang M. Ginsenosides Rb1 and $\mathrm{Rg} 1$ protect primary cultured astrocytes against oxygen-glucose deprivation/reoxygenation-induced injury via improving mitochondrial function. Int $J$ Mol Sci. 2019;20(23):6086. doi:10.3390/ ijms20236086

36. Chaunchaiyakul R, Leelayuwat N, Wu JF, Huang CY, Kuo CH. Contrasting actions of ginsenosides $\mathrm{Rb} 1$ and $\mathrm{Rg} 1$ on glucose tolerance in rats. Chin J Physiol. 2019;62(6):267-272. doi:10.4103/cjp.Cjp_61_19

37. Fernández-Moriano C, González-Burgos E, Iglesias I, Lozano R, Gómez-Serranillos MP. Evaluation of the adaptogenic potential exerted by ginsenosides $\mathrm{Rb} 1$ and $\mathrm{Rg} 1$ against oxidative stress-mediated neurotoxicity in an in vitro neuronal model. PLoS One. 2017;12(8):e0182933. doi:10.1371/journal.pone.0182933

38. Zhou P, Xie W, He S, et al. Ginsenoside Rb1 as an anti-diabetic agent and its underlying mechanism analysis. Cells. 2019;8(3):204. doi: $10.3390 /$ cells 8030204

39. Babu S, Jayaraman S. An update on $\beta$-sitosterol: a potential herbal nutraceutical for diabetic management. Biomed Pharm. 2020;131:110702. doi:10.1016/j.biopha.2020.110702

40. Wong HS, Chen JH, Leong PK, Leung HY, Chan WM, Ko KM. $\beta$ sitosterol protects against carbon tetrachloride hepatotoxicity but not gentamicin nephrotoxicity in rats via the induction of mitochondrial glutathione redox cycling. Molecules (Basel, Switzerland). 2014;19 (11):17649-17662. doi:10.3390/molecules191117649

41. Shi $\mathrm{C}, \mathrm{Wu} \mathrm{F}, \mathrm{Xu} J$. Incorporation of $\beta$-sitosterol into mitochondrial membrane enhances mitochondrial function by promoting inner mitochondrial membrane fluidity. $J$ Bioenerg Biomembr. 2013;45 (3):301-305. doi:10.1007/s10863-012-9495-3

42. Lykkesfeldt J, Svendsen O. Oxidants and antioxidants in disease: oxidative stress in farm animals. Vet $J$. 2007;173(3):502-511. doi:10.1016/j.tvj1.2006.06.005

43. Li H, Liu Y, Lin LT, et al. Acupuncture reversed hippocampal mitochondrial dysfunction in vascular dementia rats. Neurochem Int. 2016;92:35-42. doi:10.1016/j.neuint.2015.12.001

44. Thérond P, Bonnefont-Rousselot D, Davit-Spraul A, Conti M, Legrand A. Biomarkers of oxidative stress: an analytical approach. Curr Opin Clin Nutr Metab Care. 2000;3(5):373-384. doi:10.1097/ 00075197-200009000-00009

45. Cui X, Gong J, Han H, et al. Relationship between free and total malondialdehyde, a well-established marker of oxidative stress, in various types of human biospecimens. J Thorac Dis. 2018;10 (5):3088-3097. doi:10.21037/jtd.2018.05.92

46. Qin Y, Fan J, Yang W, et al. Endogenous Cys-Assisted GSH@AgNCs-rGO nanoprobe for real-time monitoring of dynamic change in GSH levels regulated by natural drug. Anal Chem. 2020;92 (2):1988-1996. doi:10.1021/acs.analchem.9b04374 
47. Lan R, Li S, Chang Q, Zhao Z. Chitosan oligosaccharides protect sprague dawley rats from cyclic heat stress by attenuation of oxidative and inflammation stress. Animals (Basel). 2019;9(12). doi:10.3390/ani9121074

48. Herzig S, Shaw RJ. AMPK: guardian of metabolism and mitochondrial homeostasis. Nat Rev Mol Cell Biol. 2018;19(2):121-135. doi:10.1038/nrm.2017.95

49. Wen F, An C, Wu X, et al. MiR-34a regulates mitochondrial content and fat ectopic deposition induced by resistin through the AMPK/ $\operatorname{PPAR} \alpha$ pathway in HepG2 cells. Int $J$ Biochem Cell Biol. 2018;94:133-145. doi:10.1016/j.biocel.2017.11.008

50. Ihsan M, Markworth JF, Watson G, et al. Regular postexercise cooling enhances mitochondrial biogenesis through AMPK and p38 MAPK in human skeletal muscle. Am J Physiol Regul Integr Comp Physiol. 2015;309(3):R286-94. doi:10.1152/ ajpregu.00031.2015

51. Buler M, Aatsinki SM, Izzi V, Uusimaa J, Hakkola J. SIRT5 is under the control of PGC-1 $\alpha$ and AMPK and is involved in regulation of mitochondrial energy metabolism. FASEB j. 2014;28(7):3225-3237. doi:10.1096/fj.13-245241

52. Kaarniranta K, Kajdanek J, Morawiec J, Pawlowska E, Blasiak J. PGC- $1 \alpha$ protects RPE cells of the aging retina against oxidative stress-induced degeneration through the regulation of senescence and mitochondrial quality control. The significance for AMD pathogenesis. Int J Mol Sci. 2018;19(8). doi:10.3390/ijms19082317

53. Calegari VC, Zoppi CC, Rezende LF, Silveira LR, Carneiro EM, Boschero AC. Endurance training activates AMP-activated protein kinase, increases expression of uncoupling protein 2 and reduces insulin secretion from rat pancreatic islets. $J$ Endocrinol. 2011;208 (3):257-264. doi:10.1530/joe-10-0450

54. Donadelli M, Dando I, Fiorini C, Palmieri M. UCP2, a mitochondrial protein regulated at multiple levels. Cell Mol Life Sci. 2014;71 (7):1171-1190. doi:10.1007/s00018-013-1407-0

55. Holley CT, Duffy CM, Butterick TA, et al. Expression of uncoupling protein-2 remains increased within hibernating myocardium despite successful coronary artery bypass grafting at 4 wk post-revascularization. J Surg Res. 2015;193(1):15-21. doi:10.1016/ j.jss.2014.08.003
56. Yuan Y, Yao YF, Hu SN, Gao J, Zhang LL. MiR-133a is functionally involved in doxorubicin-resistance in breast cancer cells MCF-7 via its regulation of the expression of uncoupling protein 2. PLoS One. 2015;10(6):e0129843. doi:10.1371/journal.pone.0129843

57. Futawaka K, Tagami T, Fukuda Y, et al. Growth hormone regulates the expression of UCP2 in myocytes. Growth Horm IGF Res. 2016;29:57-62. doi:10.1016/j.ghir.2016.04.005

58. Li J, Jiang R, Cong X, Zhao Y. UCP2 gene polymorphisms in obesity and diabetes, and the role of UCP2 in cancer. FEBS Lett. 2019;593 (18):2525-2534. doi:10.1002/1873-3468.13546

59. Fan L, Zhang C, Ai L, et al. Traditional uses, botany, phytochemistry, pharmacology, separation and analysis technologies of Euonymus alatus (Thunb.) Siebold: a comprehensive review. J Ethnopharmacol. 2020;259:112942. doi:10.1016/j.jep.2020.112942

60. Chen Z, Zhang C, Gao F, et al. A systematic review on the rhizome of Ligusticum chuanxiong Hort. (Chuanxiong). Food Chem Toxicol. 2018;119:309-325. doi:10.1016/j.fct.2018.02.050

61. Lee S, Moon E, Choi SU, Kim KH. Lignans from the Twigs of Euonymus alatus (Thunb.) siebold and their biological evaluation. Chem Biodivers. 2016;13(10):1391-1396. doi:10.1002/cbdv.201600083

62. Li N, Liu J, Zheng R, et al. Comparative study on pharmacological effects of Shenma Yizhi formula processed with three kinds of preparation techniques. Beijing J Trad Chin Med. 2019;05:427-432, 513. doi:10.16025/j.1674-1307.2019.05006

63. Zhang Y, Liu J, Yang B, et al. Ginkgo biloba extract inhibits astrocytic lipocalin-2 expression and alleviates neuroinflammatory injury via the JAK2/STAT3 pathway after ischemic brain stroke. Front Pharmacol. 2018;518. doi:10.3389/fphar.2018.00518

64. Chang S, Liu J, Liu M, et al. Experimental study on acute toxicity of Shenma Yizhi Decoction. Chin J Integr Med. 2020;03:424-426. doi:10.12102/j.issn.1672-1349.2020.03010

65. Cao Y, Jia X, Wei Y, et al. Traditional Chinese Medicine Huannao Yicong decoction extract decreases Tau hyperphosphorylation in the brain of Alzheimer's disease model rats induced by $\mathrm{A} \beta(1-42)$. Evid Based Complement Alternat Med. 2016;2016:6840432. doi:10.1155/ 2016/6840432
Neuropsychiatric Disease and Treatment

\section{Publish your work in this journal}

Neuropsychiatric Disease and Treatment is an international, peerreviewed journal of clinical therapeutics and pharmacology focusing on concise rapid reporting of clinical or pre-clinical studies on a range of neuropsychiatric and neurological disorders. This journal is indexed on PubMed Central, the 'PsycINFO' database and CAS, and is the official journal of The International Neuropsychiatric Association (INA). The manuscript management system is completely online and includes a very quick and fair peer-review system, which is all easy to use. Visit http://www.dovepress.com/testimonials.php to read real quotes from published authors. 